\title{
Neuroimmune Interactions in Sjögren's Syndrome: Relationship of Exocrine Gland Dysfunction with Autoantibodies to Muscarinic Acetylcholine Receptor-3 and Mental Health Status Parameters
}

\author{
Magdolna Deák ${ }^{a} \quad$ Attila Szvetnik $^{f} \quad$ Attila Balog $^{\mathrm{a}}$ Nicolette Sohár ${ }^{\mathrm{b}}$ \\ Renáta Varga ${ }^{c}$ Gyula Pokorny ${ }^{a}$ Gábor Tóth ${ }^{d}$ Mária Kiss ${ }^{\mathrm{e}}$ László Kovács ${ }^{a}$ \\ Departments of ${ }^{a}$ Rheumatology, ${ }^{b}$ Ophthalmology, ${ }^{c}$ Oral and Maxillofacial Surgery, ${ }^{d}$ Medical Chemistry and \\ eDermatology and Allergology, Faculty of Medicine, Albert Szent-Györgyi Health Centre, University of Szeged, and \\ ${ }^{f}$ Institute of Biotechnology, Bay Zoltán Foundation for Applied Research, Szeged, Hungary
}

\section{Key Words}

Sjögren's syndrome $\cdot$ Antimuscarinic acetylcholine receptor-3 antibody - Secretory dysfunction - Systemic lupus erythematosus $\cdot$ Rheumatoid arthritis $\cdot$ ELISA . Mental health status

\section{Abstract \\ Objectives: Antimuscarinic acetylcholine receptor-3 (m3AChR) autoantibodies have been described in primary Sjögren's syndrome (pSS). The aim of this study was to com- pare various methods for their detection and to assess the contributions of anti-m3AChR and other immunological and psychosocial factors to the pathomechanism of secondary SS (sSS). Methods: Sixty-five rheumatoid arthritis (RA) pa- tients, 103 systemic lupus erythematosus (SLE) patients, 76 pSS patients and 50 controls were compared. Three immu- nodominant epitopes of $\mathrm{m} 3 \mathrm{AChR}$ were synthesized and used in ELISA. Two extracellular epitopes were also prepared in fusion with glutathione-S-transferase and one in conjuga- tion with bovine serum albumin. Mental health status was assessed with the 36-item Short-Form Health Survey and Functional Assessment of Chronic Illness Therapy fatigue scale. Correlations were evaluated between glandular func- tion and anti-m3AChR positivities and specificities, features}

of SLE and RA, and mental health parameters. Results: Fourteen RA and 27 SLE patients had sSS. The autoantibody levels to all epitopes of $\mathrm{m} 3 \mathrm{AChR}$ were significantly higher in pSS and SLE patients than in the controls. The fusion protein forms discriminated RA from $\mathrm{pSS}$ and SLE; furthermore, the YNIP fusion protein also distinguished pSS from SLE. The prevalence and the mean levels of all autoantibodies did not differ statistically between sicca and non-sicca SLE or RA patients. Glandular dysfunction correlated with higher age in SLE and RA and an impaired health-related quality of life in SLE. Conclusions: The second and third extracellular loops of m3AChR are antigenic in pSS. Immunoassays with antigens as fusion peptides demonstrate the best performance. Sicca SLE patients have worse mental health status. Antim3AChR antibodies represent a peculiar example of neuroimmune interactions.

Copyright $\odot 2012$ S. Karger AG, Basel

\section{Introduction}

Sjögren's syndrome (SS) is an autoimmune disease with multisystem involvement. It is characterized by chronic inflammation of the exocrine glands, particularly the lachrymal and salivary glands, and a wide vari-

\section{KARGER \\ Fax +4161306 1234 \\ E-Mail karger@karger.ch}

www.karger.com
(C) 2012 S. Karger AG, Basel

$1021-7401 / 13 / 0202-0079 \$ 38.00 / 0$

Accessible online at:

www.karger.com/nim
Magdolna Deák, MD

Department of Rheumatology, Faculty of Medicine

Albert Szent-Györgyi Health Centre, University of Szeged

Kálvária sgt. 57, HU-6725 Szeged (Hungary)

E-Maildrdeakmagdi@gmail.com 
ety of further immune-mediated organ involvement [1]. In the affected secretory tissues, focal lymphocytic infiltration can be observed. The fundamental symptoms are decreased tear and saliva production, which lead to keratoconjunctivitis sicca and xerostomia, respectively. SS often presents as a secondary condition, when it overlaps with various systemic autoimmune diseases; in this case it is termed secondary SS (sSS), in contrast with primary SS (pSS) as a separate entity.

It has previously been observed that the extent of salivary gland damage caused by lymphocytic infiltration is not proportional to the salivary flow impairment [2]. This indicates the role of other underlying mechanisms, such as the functional inhibition of fluid secretion. It is well known that the autonomic nervous system modulates saliva production through the functionally dominant subtype 3 muscarinic acetylcholine receptor (m3AChR) on acinar cells. An immune-mediated mechanism has been hypothesized to alter the glandular function in the target organ by means of autoantibodies which block the postsynaptic muscarinic receptors [3-5].

Indeed, we and several other groups have demonstrated the presence of circulating autoantibodies directed against the m3AChR in patients with pSS [6-12], and their binding to muscarinic receptors on human salivary gland acinar cells has also been confirmed [13]. Although several animal models and human data support the importance of anti-m $3 \mathrm{AChR}$ antibodies in pSS, these data mostly originate from functional assays [14-17], and a validated immunodiagnostic test is still not available. The exact epitope specificity of anti-m $3 \mathrm{AChR}$ has also remained unidentified, which hampers their detection. Moreover, the prevalence and pathogenetic role of anti$\mathrm{m} 3 \mathrm{AChR}$ antibodies in sSS have not been systematically examined.

The pathogenesis of sSS is heterogeneous and not completely clear. The histological alterations in the salivary glands of sSS overlapping various systemic autoimmune diseases are variable, as is the clinical picture [1820]. Sicca symptoms are common not only in definite SS, but also in fibromyalgia, chronic fatigue syndrome and various other noninflammatory chronic diseases $[21,22]$. In these conditions, chronic stress and an altered neuroendocrine homeostasis have been hypothesized as causes of the exocrine insufficiency, modulated by an autonomic nervous system dysfunction.

The present study had two major objectives. First, we wished to determine the antigenic epitope of $\mathrm{m} 3 \mathrm{AChR}$ which interacts with autoantibodies from pSS, systemic lupus erythematosus (SLE) and rheumatoid arthritis
(RA) patients and also to assess the presence and clinical correlates of the receptor-specific autoantibodies in the studied disease groups. Accordingly, we attempted to develop an appropriate immunodiagnostic method for the detection of these antibodies. As sSS is an entity with a complex pathogenesis, our second objective was to address the relative contributions of immunological factors, and in particular anti-m3AChR antibodies, and mental health status to the elicitation of the sicca complex arising in $\mathrm{pSS}$, SLE and RA.

\section{Patients and Methods}

\section{Patients}

Data on 65 patients with RA and 103 with SLE were compared with those on $76 \mathrm{pSS}$ patients and 50 healthy controls. The disease groups were classified according to the appropriate international criteria, namely the 2010 RA criteria of the American College of Rheumatology/European League against Rheumatism [23], the modified 1982 SLE criteria of the American College of Rheumatology [24] and the 2002 American-European Consensus Criteria (AECC) for SS [25]. The mean age of the RA patients (11 male and 54 female) was 58 years (22-82); that of the SLE patients (9 male and 94 female) was 48 years (25-74), and that of the pSS patients (2 male and 74 female) was 56 years (30-76).

All the RA and SLE patients were systematically evaluated with regard to the presence of a sicca complex; following the AECC for SS, subjective sicca complaints were regarded as present if at least 1 of the 3 questions included in these criteria was answered positively, while an objective exocrine deficiency was evaluated by means of the Schirmer test and the measurement of unstimulated whole saliva production. Sicca complex was established if subjective sicca symptoms of both the eye and the mouth were associated with at least 1 positive objective test on at least 2 separate occasions. Determination of the presence of sSS as classified by the AECC was also attempted, but since labial salivary gland biopsy was not performed in several patients for ethical reasons, these assessments are available for only a proportion of the patients.

The protocol was approved by the Human Investigation Review Board of the University of Szeged, Faculty of Medicine, and the study was performed in accordance with the principles of the Declaration of Helsinki. A variety of disease-specific clinical and immunoserological parameters of the patients were collected, and selected clinical and laboratory variables, including the immunoserological profile of SLE and RA, are presented in table 1.

The impact of immunosuppressive and anticholinergic medication on the exocrine function was assessed. Therapies with proven anticholinergic effect were evaluated, including antihistaminic, antidepressant, antipsychotic, antispasmodic, antiemetic and antiepileptic drugs. RA and SLE patients were on the usual immunosuppressive therapies, mostly low-dose corticosteroid, methotrexate, leflunomide, chloroquine, rarely sulphasalazine, azathioprine, cyclosporin A, cyclophosphamide, mycophenolate mofetil, tumor necrosis factor- $\alpha$ inhibitor or anti-CD20 biologic agents. Treatment with none of the studied medications showed a correlation with the presence of sicca complex (data not shown). 
Table 1. Characteristic clinical and laboratory variables of SLE $(\mathrm{n}=103)$ and RA patients $(\mathrm{n}=65)$

\begin{tabular}{lllr}
\hline SLE $(\mathrm{n}=103)$ & $\%$ & RA $(\mathrm{n}=65)$ & $\%$ \\
\hline Polyarthritis & 89.2 & Rheumatoid nodules & 12.5 \\
Photosensitivity & 70.6 & Pulmonary fibrosis & 4.7 \\
Nephritis & 30.4 & RF & 84.4 \\
Serositis & 28.4 & Anti-MCV & 77.9 \\
Nervous system & 21.6 & Anti-SSA & 9.5 \\
Anti-dsDNA & 74.2 & Anti-SSB & 7.1 \\
Anti-SSA & 50.5 & & \\
Anti-SSB & 38.6 & & \\
Low C3, C4 & 67.6 & & \\
\hline
\end{tabular}

Anti-dsDNA = Antibody to double-stranded DNA; antiSSA = anti-Sjögren's syndrome A; anti-SSB = anti-Sjögren's syndrome B; C3 = complement -3 ; C4 = complement 4 ; RF = rheumatoid factor; anti-MCV = antimutant citrullinated vimentin.

\section{Antigen Preparation}

Three immunodominant epitopes of the human m3AChR were predicted by means of the computer software Peptide Companion version 1.231 (Coshisoft/PeptiSearch); peptide amino acids (aa) 184-227 (AGSE) from the second extracellular loop of the receptor, where the ligand binding region is localized, peptide aa 506-521 (YNIP) from the third extracellular loop and peptide aa 360-377 (TRIC) from the third intracellular loop were selected as antigens.

Multiple forms of these antigens were prepared for ELISA to test whether modification of $\mathrm{m} 3 \mathrm{AChR}$ peptides could enhance the antigenicity and improve the detection of the autoantibodies specific to the respective peptides. First, short linear peptides were synthesized with solid-phase peptide synthesis as described previously [6]. We then constructed recombinant fusion proteins containing the peptides AGSE and YNIP fused with glutathione$S$-transferase (GST), because it was our previous experience that this protein microenvironment can enhance the assumption of an appropriate physiological conformation [26]. Briefly, peptidecoding DNA sequences were assembled from synthetic oligonucleotides and cloned into expression vectors in fusion with GST; the fusion product was expressed in Escherichia coli and purified by affinity chromatography [for details, see 6]. Finally, the epitope with the highest predicted antigenicity (AGSE) was also prepared in multiple conjugation to bovine serum albumin (BSA) in an attempt to enhance the sensitivity of the assay. We compared the performances of the ELISA systems by using the different forms of the antigens.

\section{ELISA Techniques}

Microtiter plates were coated with the above-mentioned antigens (AGSE: $1 \mu \mathrm{g} / \mathrm{ml}$; YNIP and TRIC synthetic peptides: $2 \mu \mathrm{g} /$ $\mathrm{ml}$; GST fusion products and BSA-conjugated antigen: $10 \mu \mathrm{g} / \mathrm{ml}$ ). After incubation overnight at $4{ }^{\circ} \mathrm{C}$, patient sera were added at a dilution of 1:200 in PBS-Tween followed by peroxidase-labeled antihuman IgG (1:2,500 in PBS-Tween; Sigma-Aldrich Hungary, Budapest). Optical density (OD) was read at 492 and $620 \mathrm{~nm}$ after the addition of ortho-phenylenediamine to the samples. Measurements were made in duplicate, and appropriate numbers of negative controls were used for every microtiter plate. For the GST- or BSA-conjugated proteins, the specific corrected OD characterizing the $\mathrm{m} 3 \mathrm{AChR}$-specific epitope within an individual sample was calculated by subtracting the OD for the GST or BSA protein from that for the GST-m3AChR peptide fusion protein or BSAAGSE conjugate. As the absolute OD values for the negative controls varied from plate to plate, the OD values of the patients' samples were normalized to the mean of the controls for every plate by dividing the OD of the test sample by the cutoff (mean $+2 \mathrm{SD}$ of the negative control OD values). The resulting relative OD values were used for the calculations.

\section{Mental Health Status Assessments}

Physical and mental health were assessed with the following validated questionnaires: the 36-item Short-Form Health Survey (SF-36) and the Functional Assessment of Chronic Illness Therapy (FACIT) fatigue scale $[27,28]$. The SF-36 consists of 36 questions which represent multiple indicators of mental and physical health, including behavioral function and dysfunction, distress and well-being, objective reports and subjective ratings, and both favorable and unfavorable self-evaluations of general health status [27]. The FACIT fatigue scale is a short, 13-item tool that measures an individual's level of fatigue during the usual daily activities over the past week.

\section{Statistical Methods}

The differences in mean autoantibody concentrations between the studied groups were assessed with analysis of variance with Bonferroni's correction as a post hoc test. The occurrences of positivities to the various antigenic epitopes were compared between the different patient and control groups with the $\chi^{2}$ test. The demographic, clinical and immunoserological parameters, the levels of anti-m $3 \mathrm{AChR}$ antibodies specific to the various antigens and the SF-36 and FACIT results were compared between the RA and SLE patients with or without sicca complex through use of the Student $t$ test or Fisher's exact test, as appropriate, depending on whether continuous or categorical variables were tested. Levels of $\mathrm{p}<0.05$ were taken as statistically significant. The statistical analyses were performed with SPSS 15.0 software.

\section{Results}

\section{Anti-m3AChR Detection}

The mean levels of anti-m3AChR antibodies depending on the method of antigen preparation and epitope specificity in the three disease groups and the healthy controls can be seen in figure 1. Significantly higher mean autoantibody levels to the peptide sequences corresponding to the second (AGSE) and third (YNIP) extracellular loops were detected in both the pSS and the SLE patients than in the controls, while the RA patients exhibited only a borderline significant difference $(\mathrm{p}=$ 0.067 ) versus controls. This technique using short peptide sequences failed to make further distinctions be- 
Fig. 1. Mean relative OD values of antim3AchR antibodies measured with ELISA. The mean values of all studied antim3AChR antibodies were significantly higher in both pSS and SLE patients than in the controls. GST-AGSE also discriminated $\mathrm{pSS}$ and SLE from RA. ${ }^{\mathrm{a}} \mathrm{p}<0.05$ versus control; ${ }^{b} \mathrm{p}<0.05$ versus RA. GAGSE = GST-AGSE fusion peptide; GYNIP = GST-YNIP fusion peptide; BAGSE = BSAAGSE multiple-conjugated peptide.

Fig. 2. The prevalences of anti-m $3 A C h R$ antibody positivities in the three disease groups and the controls. The prevalence of GST-YNIP showed a significant difference between pSS and the other groups. GSTAGSE discriminated pSS and SLE from RA and the controls. ${ }^{\mathrm{a}} \mathrm{p}<0.05$ versus control; ${ }^{b} \mathrm{p}<0.05$ versus $\mathrm{RA} ;{ }^{\mathrm{c}} \mathrm{p}<0.05$ versus SLE. GAGSE = GST-AGSE fusion peptide; GYNIP = GST-YNIP fusion peptide; BAGSE = BSA-AGSE multiple-conjugated peptide.
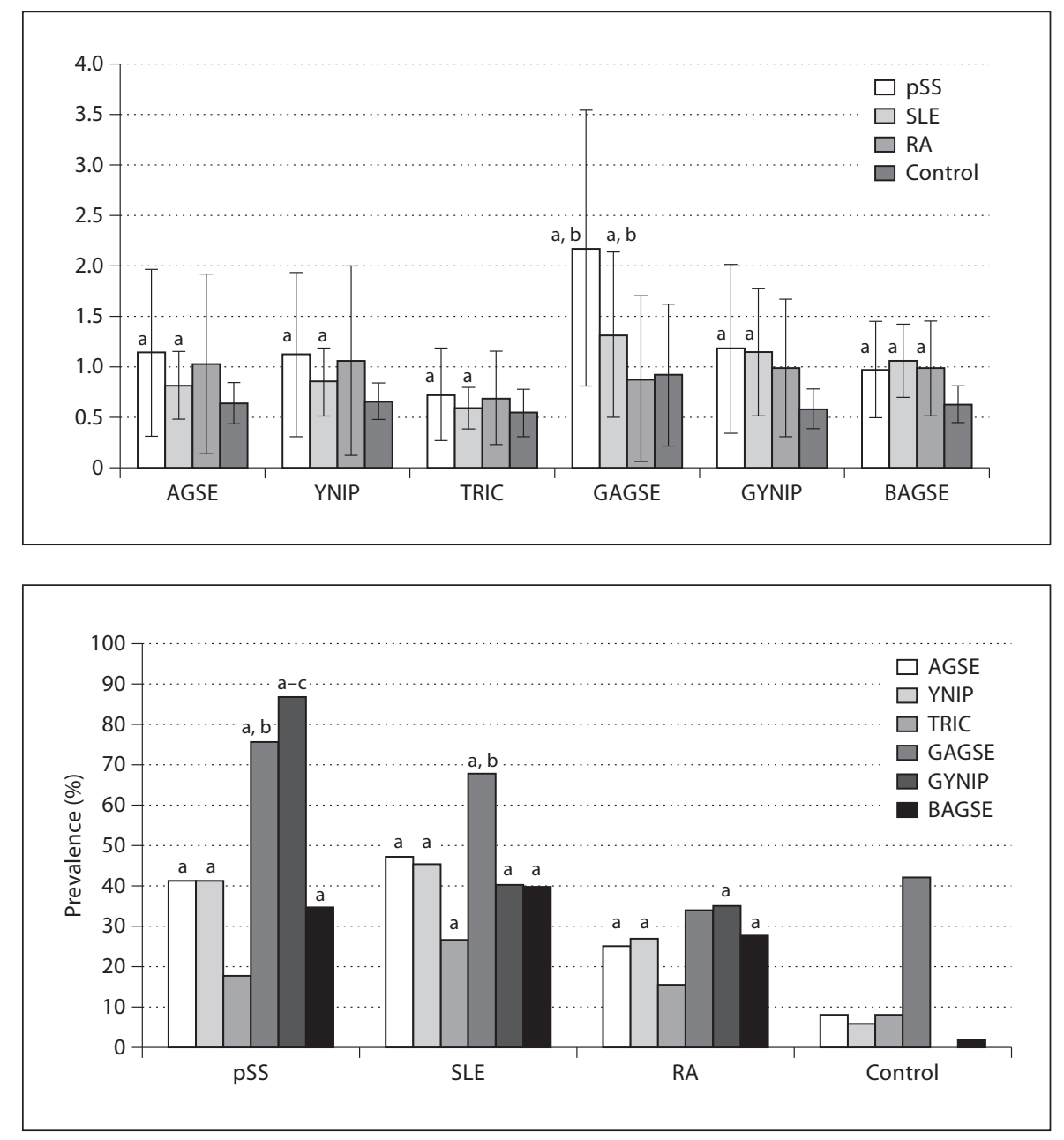

tween the pSS, SLE and RA patients. When the AGSE peptide was presented in fusion with GST, this form displayed significantly lower mean antibody levels in RA as compared with the other two patient groups. ELISA using the BSA conjugate failed to identify antibodies with sufficient group-discriminative power, although the mean autoantibody levels to this antigen were higher in all three diseases than in the controls. It also became evident that the TRIC peptide, which is located in the intracellular portion of the $\mathrm{m} 3 \mathrm{AChR}$, reacted with only a small proportion of the sera and therefore cannot be regarded as antigenic in these diseases.

An assessment of the prevalence of the studied antibodies confirmed and further clarified the results (fig. 2). Autoantibodies to the short peptide sequences of AGSE and YNIP occurred at significantly higher frequencies in all disease groups than in the controls. Furthermore, the use of GST fusion forms also revealed differences be- tween the patient groups; the prevalences of GST-AGSE and GST-YNIP were elevated in both pSS and SLE as compared with RA. Furthermore, antibodies to GSTYNIP were also able to discriminate between pSS and SLE patients, as these antibodies occurred at significantly higher frequency in pSS than in SLE, though in SLE they were still significantly more prevalent than in RA or in the controls. In summary, short peptide sequences detected anti-m3AchR with relatively high specificity but low sensitivity, whereas fusion with GST preserved the specificity and enhanced the sensitivity of detection of anti-GST-YNIP. Multiple peptide conjugation to BSA did not improve the sensitivity of the ELISA (table 2).

\section{Clinical Correlations}

In the second part of the study, we sought clinical, immunological and psychosomatic correlates of the sicca complex arising in SLE or RA patients. Sicca symptoms 
Table 2. Sensitivity and specificity of the tests for the detection of anti-m3AChR antibodies to discriminate between pSS and healthy controls

\begin{tabular}{llc}
\hline & Sensitivity & Specificity \\
\hline AGSE & $41.2 \%$ & $92.0 \%$ \\
YNIP & $41.2 \%$ & $94.0 \%$ \\
TRIC & $17.6 \%$ & $92.0 \%$ \\
GAGSE & $75.6 \%$ & $58.1 \%$ \\
GYNIP & $86.5 \%$ & $100.0 \%$ \\
BAGSE & $34.7 \%$ & $98.2 \%$ \\
\hline
\end{tabular}

ELISA with the short synthetic peptide sequences had high specificity but low sensitivity in the detection of anti-m $3 A C h R$. Fusion with GST preserved the specificity and enhanced the sensitivity of the detection of antibodies to the YNIP sequence. Multiple peptide conjugation to BSA did not improve detection. GAGSE = GST-AGSE fusion peptide; GYNIP = GST-YNIP fusion peptide; BAGSE = BSA-AGSE multiple-conjugated peptide.

Table 3. Differences in the clinical features and selected SF-36 items of the sicca and non-sicca SLE patients

\begin{tabular}{lcll}
\hline & $\begin{array}{l}\text { Non-sicca } \\
(\mathrm{n}=76)\end{array}$ & $\begin{array}{l}\text { Sicca } \\
(\mathrm{n}=27)\end{array}$ & $\mathrm{p}$ \\
\hline $\begin{array}{l}\text { Clinical features, \% } \\
\text { Organic brain syndrome }\end{array}$ & 1.3 & 11.1 & 0.056 \\
$\begin{array}{l}\text { Stroke } \\
\text { Anti-SSA }\end{array}$ & 0 & 7.4 & 0.068 \\
Nephritis & 45.9 & 62.9 & 0.098 \\
Lymphopenia & 34.6 & 18.5 & 0.091 \\
\hline SF-36, mean values & 57.3 & 37 & 0.056 \\
Role-physical & 39.33 & 35.05 & 0.081 \\
Vitality & 46.71 & 41.70 & $\mathbf{0 . 0 0 5}$ \\
Social functioning & 42.68 & 38.04 & $\mathbf{0 . 0 4 1}$ \\
Mental health & 42.12 & 36.82 & $\mathbf{0 . 0 4 8}$ \\
Mental composite score & 44.01 & 38.52 & $\mathbf{0 . 0 3 5}$ \\
\hline
\end{tabular}

Higher SF-36 mean values represent better health status.

Significant differences are shown in bold. Anti-SSA = antiSjögren's syndrome A.

were common in both SLE and RA patients; the sicca complex was verified in 14 RA patients $(21.5 \%)$ and 27 SLE patients (26.2\%). We compared the sicca and nonsicca patients with regard to the aspects of age, disease duration, disease-specific organ involvement, immunoserology and mental health status parameters, as well as antimuscarinic receptor antibody positivity and epitope

Neuroimmune Interactions in Sjögren's Syndrome specificity. Decreased saliva production correlated with increasing age in both SLE and RA. Furthermore, in the SLE patients with sicca complex, organic brain syndrome, stroke and anti-SSA antibody positivity occurred more frequently, whereas nephritis and lymphopenia were seen more rarely than in the non-sicca SLE patients, all with borderline significance (table 3 ). It is noteworthy that the SLE patients with sicca syndrome demonstrated significantly impaired mental health parameters on the SF-36, specifically on the 'role-physical', vitality, social function and mental health domains and the composite mental score, as compared with the non-sicca SLE patients (table 3). Conversely, the FACIT fatigue scale did not reveal differences between the subgroups of sicca and non-sicca SLE patients. In contrast, in the RA patients, no clinical or immunological correlates other than increasing age were identified that could discriminate between those with or without sicca complex; moreover, none of the parameters that characterize quality of life (SF-36) or fatigue (FACIT) were found to correlate with the presence of sicca syndrome. Finally, although anti-m $3 A$ ChR antibodies specific to the second or third extracellular loops occurred in both SLE and RA patients, the prevalence and the mean levels of these autoantibodies were not statistically different between the patient subgroups of SLE or RA with or without sicca complex.

\section{Discussion}

This study has revealed two distinct neuroimmune interactions that may contribute to the complex pathophysiology of pSS and sSS, namely autoantibody production that directly targets autonomic neurotransmitter receptors, specifically $\mathrm{m} 3 \mathrm{AChR}$, and an altered mental health status that may theoretically be regarded as a central inhibitory mechanism of the parasympathetic innervation of the salivary glands.

Anti-m3AChR autoantibodies have been hypothesized to bind to the m3AChR and block the parasympathetic neurotransmission $[5,14]$, thereby serving as an example of how a pathological immunological process can lead to organ dysfunction by inhibiting the normal function of the autonomic nervous system. Although several experimental settings, involving rodent tissues and cultured human salivary glands, have supported this concept, formal proof of the functional role of anti$\mathrm{m} 3 \mathrm{AChR}$ antibodies in humans is still lacking because the reliable detection of these autoantibodies has remained elusive. Convincing correlations between anti- 
muscarinic receptor antibodies in SS and other systemic autoimmune diseases could therefore not be addressed.

We previously developed an ELISA assay involving the second extracellular loop of the human m $3 A C h R$ as antigen and succeeded in demonstrating anti-m $3 \mathrm{AChR}$ antibodies in the majority of pSS patients $[6,7]$. Other investigators have also subsequently proved the presence of these autoantibodies, with frequencies in a rather wide range, and even proposed further epitopes on the $\mathrm{m} 3 \mathrm{AChR}$ as the targets of the immune response in pSS, specifically on the third extracellular loop [10-12, 14, 28]; however, others have failed to confirm our results $[17,29$, 30]. Furthermore, the role of anti-m $3 \mathrm{AChR}$ antibodies in sSS, which occurs in a large proportion of patients with two common systemic autoimmune diseases, SLE and RA, has not been examined to date.

The results of the present study appear to confirm our previous findings that the second extracellular loop is antigenic in pSS and that the GST fusion construct is an appropriate mode of antigen presentation [26]. In addition, our findings support the recent results that the third extracellular loop also contains antigenic sequences in a considerable proportion of pSS patients (and also in some SLE and RA patients). Actually, anti-GST-YNIP has emerged as the diagnostically most useful antibody, as it discriminates pSS patients from controls with the best balance of specificity and sensitivity, and in pSS, these autoantibodies occurred more frequently not only than in the controls or the RA patients but also than in SLE. Of course, this apparently better diagnostic performance may be independent of the putative functional significance of the autoantibodies with various epitope specificities, and it should be noted that most of the previous results, including our own findings that directly visualized the binding of anti-m $3 \mathrm{AChR}$ to the receptor in human salivary glands, favored the inhibitory role of antimAChR targeting the second extracellular loop, where the ligand-binding region is located. Therefore, we conclude that further validation of these results on larger patient populations and potentially more precise epitope mapping are needed to elucidate the exact epitope specificity and functional importance of these autoantibodies. Moreover, our data provide no indication as to whether anti-m3AChR antibodies may be related to the pathogenesis of sSS overlapping SLE or RA, although the examined patient population may well have been too small for definite conclusions to be drawn.

Sicca symptoms often develop in chronic illnesses other than pSS. The pathogenesis of sSS overlapping different systemic autoimmune diseases, including SLE, RA or scleroderma, is rather variable. An associated sSS alters the immunological phenotype of SLE, including the organ manifestations or the immunoserologic profile [19, 31]. Some studies have suggested that the probability of the development of sicca symptoms in RA is directly correlated with the cumulative disease activity and the degree of functional impairment, i.e. the burden of the chronic disease [32], while others did not find such associations [33]. A sicca syndrome clinically indistinguishable from SS also develops in a considerable proportion of patients with organ-specific autoimmune diseases, such as primary biliary cirrhosis, multiple sclerosis or gluten-sensitive enteropathy [34], and in other chronic non-immune-mediated illnesses, such as fibromyalgia or chronic fatigue syndrome [35]. Another example of such a nonautoimmune sicca syndrome is the 'sicca, asthenia, polyalgia syndrome'. It was revealed by Champey et al. [22] that the degree of deterioration of the quality of life - measured with the SF-36 as used in this study - directly correlated with the presence and severity of sicca symptoms in this syndrome. We therefore set out to examine whether mental health alterations, assessed with the SF-36 and the FACIT fatigue scale, may be associated with the development of a sicca syndrome in SLE or RA patients.

Through the investigation of considerable numbers of SLE and RA patients, we have obtained new information about the correlates of the development of sicca syndrome in these diseases, mostly in SLE. Some clinical and immunoserological features differed between SLE patients with sicca syndrome and those without it, with borderline significance. This is very similar to the findings of Manoussakis et al. [19] in a larger cohort of patients and to the results of a recent meta-analysis [36] and justifies the assessment of sicca syndrome in our SLE cohort. Moreover, the SLE plus sicca syndrome patients in this study were clearly different from those SLE patients in whom sicca symptoms were not present, in terms of a significantly higher occurrence of signs of psychological dysfunction, specifically lower vitality, a worse perception of their mental health, difficulties in physical functions reflecting the extent to which health interferes with usual daily activities such as work, studies or household duties, and worse scores on the composite index, which indicates a lower overall level of mental well-being as compared with the SLE patients without sicca symptoms. We have found only one publication in which health-related quality of life parameters were compared between SLE patients with or without sicca symptoms, and the findings of that study are similar to our own results in 
that a lower perceived health-related status is correlated with salivary dysfunction [37]. Our results argue in favor of an association between the health-related quality of life parameters and the evolution of sicca symptoms. These mental alterations may be either consequences or causes of the sicca symptoms, or they may have a common background. Our study design is obviously not sufficient to allow an exploration of causal relationships, but some data do suggest that chronic stress has a direct impact on saliva production and may lead to chronic xerostomia $[38,39]$. Salivary levels of cortisol and chromogranin A have been found to be increased in subjects with low salivary flow rates [40]. Profound alterations in neurotransmitter homeostasis in SS were long ago observed by Santavirta et al. [41]; both the vasoactive intestinal peptide and the neuropeptide Y contents (markers of parasympathetic and sympathetic nervous system activity, respectively) in the saliva were found to differ from those in healthy subjects. As indicated by the results of a number of other investigations $[42,43]$, it is reasonable to hypothesize that chronic stress, depression and several further features of the long-standing distress associated with a systemic autoimmune illness such as SLE [44] may negatively influence the salivary gland function and are factors that should be addressed during the care of these patients.

One limitation of our study is that many of the SLE and RA patients with sicca syndrome did not strictly meet the criteria of sSS. This is mostly explained by the fact that very few patients underwent labial salivary gland biopsy once the diagnosis of SLE and RA had been verified, mostly because of patient refusal, anticoagulant therapy or the lack of specific therapeutic consequences. However, it should be emphasized that all the sicca syndrome patients consequently gave positive answers to the sicca questionnaire on at least two separate occasions and had markedly and repeatedly low salivary and lachrymal flow rates. The variability of these test results was low (data not shown), and these patients could prudently be regarded as patients with sSS in clinical practice.

In summary, the results of the present study indicate that a probably polyclonal autoimmune reaction to multiple epitopes on the m3AChR is observed most prominently in pSS, but also in SLE and RA, and the fusion of these antigenic peptides to GST may - after further validation - be a promising laboratory test for further research and possibly for the diagnosis of pSS. Furthermore, an impaired level of psychosocial well-being and a dysfunctional stress response may be contributors to the pathogenesis of sSS in SLE.

\section{Acknowledgments}

L.K. is supported by a Bolyai János Scholarship from the Hungarian Academy of Sciences. The authors are grateful to Szivia Pördi for her excellent laboratory assistance.

\section{References}

$>1$ Jonsson R, Haga HJ, Gordon TP: Current concepts on diagnosis, autoantibodies and therapy in Sjögren's syndrome. Scan J Rheumatol 2000;29:341-348.

-2 Andoh Y, Shimura S, Sawai T, Sasaki H, Takishima T, Shirato K: Morphometric analysis of airways in idiopathic pulmonary fibrosis patients with mucous hypersecretion. Am Rev Respir Dis 1993;148:1358-1362.

3 Bacman S, Sterin-Borda L, José Camusso J, Arana R, Hubscher O, Borda E: Circulating antibodies against rat parotid gland M3 muscarinic receptors in primary Sjögren's syndrome. Clin Exp Immunol 1996;14:454459.

$>4$ Robinson CP, Brayer J, Yamachika S, Esch TR, Peck AB, Stewart CA, et al: Transfer of human serum IgG to nonobese diabetic $\operatorname{Ig} \mu^{\text {null }}$ mice reveals a role for autoantibodies in the loss of secretory function of exocrine tissues in Sjögren's syndrome. Proc Natl Acad Sci USA 1998;95:7538-7543.
5 Dawson LJ, Stanbury J, Venn N, Hasdimir B, Rogers SN, et al: Antimuscarinic anbitodies in primary Sjögren's syndrome reversibly inhibit the mechanism of fluid secretion by human submandibular salivary acinar cells. Arthritis Rheum 2006;54:1165-1173.

6 Marczinovits I, Kovács L, György A, Tóth GK, Dorgai L, Molnár J, et al: A peptide of muscarinic acetylcholine receptor-3 is antigenic in primary Sjögren's syndrome. J Autoimmun 2005;24:47-54.

7 Kovács L, Marczinovits I, György A, Tóth GK, Dorgai L, Pál J, et al: Clinical associations of autoantibodies to human muscarinic receptor- $3^{213-228}$ in primary Sjögren's syndrome. Rheumatology 2005;44:1021-1025.

8 Bacman S, Berra A, Sterin-Borda L, Borda E: Muscarinic acetlycholine receptor antibodies as a new marker of dry eye Sjögren's syndrome. Invest Ophthalmol Vis Sci 2001;42: 321-327.
Zigon P, Hocevar A, Cucnik S. Bozic B, Rozman B, et al: Antibodies against 25-mer synthetic peptide of M3 muscarinic acetylcholine receptor. Arthritis Res Ther 2004;6 (suppl 1):5.

- 10 Naito Y, Matsumoto I, Wakamatsu E, Goto D, Sugiyama T, Matsumura R, et al: Muscarinic acetylcholine receptor autoantibodies in patients with Sjögren's syndrome. Ann Rheum Dis 2005;64:510-511.

$\checkmark 11$ Koo NY, Li J, Hwang SM, Choi SY, Lee SJ, Oh $\mathrm{SB}$, et al: Functional epitope of muscarinic type 3 receptor which interacts with autoantibodies from Sjögren's syndrome patients. Rheumatology 2008;47:828-833.

-12 Tsuboi H, Matsumoto I, Wakamatsu E, Nakamura Y, Izuka M, Hayashi T: New epitopes and function of anti-M3 muscarinic acetylcholine receptor antibodies in patients with Sjögren's syndrome. Clin Exp Immunol 2010;162:53-61. 
13 Kovács L, Fehér E, Bodnár I, Marczinovits I, Nagy GM, Somos J, et al: Demonstration of autoantibody binding to muscarinic acetylcholine receptors in the salivary gland in primary Sjögren's syndrome. Clin Immunol 2008;128:269-276.

14 Li J, Ha YM, Kü NY, Choi SY, Lee SJ, et al: Inhibitory effects of autoantibodies on the muscarinic receptors in Sjögren's syndrome. Lab Invest 2004;84:1430-1438.

15 Waterman SA, Gordon TP, Rischmueller M: Inhibitory effects of muscarinic receptor autoantibodies on parasympathetic neurotransmission in Sjögren's syndrome. Arthritis Rheum 2000;43:1647-1654.

-16 Park K, Haberberger RV, Gordon TP, Jackson MW: Antibodies interfering with the type 3 muscarinic receptor pathway inhibit gastrointestinal motility and cholinergic neurotransmission in Sjögren's syndrome. Arthritis Rheum 2011;63:1426-1434.

-17 Roescher N, Kingman A, Shirota Y, Chiorini JA, Illei GG: Peptide-based ELISAs are not sensitive and specific enough to detect muscarinic receptor type 3 autoantibodies in serum from patients with Sjögren's syndrome. Ann Rheum Dis 2011;70:235-236.

18 Brun JG, Madland TM, Jonsson R: A prospective study of sicca symptoms in patients with rheumatoid arthritis. Arthritis Rheum 2003;49:287-290.

-19 Manoussakis MN, Georgipoulou C, Zintzaras E, Spyropoulou M, Stavropoulou A, Skopouli FN, Moutsopoulos HM: Sjögren's syndrome associated with systemic lupus erythematosus. Arthritis Rheum 2004;50: 882-891.

20 Avouac J, Sordet C, Depinay C, et al: Systemic sclerosis-associated Sjögren's syndrome and relationship to the limited cutaneous subtype: results of a prospective study of sicca syndrome in 133 consecutive patients. Arthritis Rheum 2006;54:2243-2249.

-21 Rhodus NL, Fricton J, Carlson P, Messner R: Oral symptoms associated with fibromyalgia syndrome. J Rheumatol 2003;30:18411845.

-22 Champey J, Corruble E, Böttenberg JE, Buhl C, Meyer T, Caudmont C, Bergé E, Pellet J, Hardy P, Mariette X: Quality of life and psychological status in patients with primary Sjögren's syndrome and sicca symptoms without autoimmune features. Arthritis Rheum 2006;55:451-457.

23 Vitali C, Bombardieri S, Jonsson R, et al: Classification criteria for Sjögren's syndrome: a revised version of the European criteria proposed by the American-European Consensus Group. Ann Rheum Dis 2002;61: 554-558.
24 Hochberg MC: Updating the American College of Rheumatology revised criteria for the classification of systemic lupus erythematosus. Arthritis Rheum 1997;40:1725.

25 Aletaha D, Neogi T, Silman AJ, Funovits J, Felson DT, et al: 2010 Rheumatoid arthritis classification criteria: an American College of Rheumatology/European League Against Rheumatism collaborative initiative. Arthritis Rheum 2010;62:2569-2581.

26 Laczkó I, Vass E, Tóth GK, Marczinovits I, Kiss M, Husz S, et al: Conformational consequences of coupling bullous pemphigoid antigenic peptides to glutathione-S transferase and their diagnostic significance. J Pept Sci 2000;6:378-386.

27 McHorney CA, Ware JE Jr, Raczek AE: The MOS 36-item short form health survey (SF36). II. Psychometric and clinical tests of validity in measuring physical and mental health constructs. Med Care 1993;31:247263.

28 He J, Guo JP, Ding Y, Li YN, Pan SS, Liu Y, Li ZG: Diagnostic significance of measuring antibodies to cyclic type 3 muscarinic acetylcholine receptor peptides in primary Sjögren's syndrome. Rheumatology (Oxford) 2011;50:879-884.

29 Cavill D, Waterman SA, Gordon TP: Failure to detect antibodies to extracellular loop peptides of the muscarinic M3 receptor in primary Sjögren's syndrome. J Rheumatol 2002;29:6-8

30 Dawson LJ, Allison HE, Stanbury J, Fitzgerald D, Smith PM: Putative anti-muscarinic antibodies cannot be detected in patients with primary Sjögren's syndrome using conventional immunological approaches. Rheumatology 2004;43:1488-1495.

31 Szántó A, Szodoray P, Kiss E, Kapitány A, Szegedi G, Zeher M: Clinical, serologic, and genetic profiles of patients with associated Sjögren's syndrome and systemic lupus erythematosus. Hum Immunol 2006;67:924930.

32 Uhlig T, Kvien TK, Jensen JL, Axéll T: Sicca symptoms, saliva and tear production, and disease variables in 636 patients with rheumatoid arthritis. Ann Rheum Dis 1999;58: 415-422.

33 Haga HJ, Naderi Y, Moreno AM, Peen E: A study of the prevalence of sicca symptoms and secondary Sjögren's syndrome in patients with rheumatoid arthritis, and its association to disease activity and treatment profile. Int J Rheum Dis 2012;15:284-288.
34 von Bültzingslöwen I, Sollecito TP, Fox PC, Daniels T, Jonsson R, et al: Salivary dysfunction associated with systemic diseases: systematic review and clinical management recommendations. Oral Surg Oral Med Oral Pathol Oral Radiol Endod 2007;103(suppl 1):S57.e1-S57.e15.

35 Price EJ, Venables PJ: Dry eyes and mouth syndrome - a subgroup of patients presenting with sicca symptoms. Rheumatology (Oxford) 2002;41:416-422.

36 Yao Q, Altman RD, Wang XG: Systemic lupus erythematosus with Sjögren syndrome compared to systemic lupus erythematosus alone: a meta-analysis. J Clin Rheumatol 2012;18:28-32.

37 Gilboe IM, Kvien TK, Uhlig T, Husby G: Sicca symptoms and secondary Sjögren's syndrome in systemic lupus erythematosus: comparison with rheumatoid arthritis and correlation with disease variables. Ann Rheum Dis 2001;60:1103-1109.

38 Moret S, Coudert JL, Bejat C, Robin O, Lissac $\mathrm{M}$ : The influence of basal anxiety on unstimulated parotid and submandibular saliva. Arch Oral Biol 1993;38:751-754.

39 Bergdahl M, Bergdahl J: Low unstimulated salivary flow and subjective oral dryness: association with medication, anxiety, depression, and stress. J Dent Res 2000;79:16521656.

-40 Shigeyama C, Ansai T, Awano S, Soh I, Yoshida A, Hamasaki T, Kakinoki Y, Tominaga K, Takahashi T, Takehara T: Salivary levels of cortisol and chromogranin A in patients with dry mouth compared with age-matched controls. Oral Surg Oral Med Oral Pathol Oral Radiol Endod 2008;106:833-839.

-41 Santavirta N, Konttinen YT, Törnwall J, Segerberg M, Santavirta S, Matucci-Cerinic M, Björvell $\mathrm{H}$ : Neuropeptides of the autonomic nervous system in Sjögren's syndrome. Ann Rheum Dis 1997;56:737-740.

-42 Hill CM, Walker RV: Salivary cortisol determinations and self-rating scales in the assessment of stress in patients undergoing the extraction of wisdom teeth. Br Dent J 2001; 191:513-515.

43 Rivera Gómez B, Hernández Vallejo G, Arriba de la Fuente L, López Cantor M, Diaz M, López-Pintor RM: The relationship between the levels of salivary cortisol and the presence of xerostomia in menopausal women. A preliminary study. Med Oral Patol Oral Cir Bucal 2006;101:E407-E412.

44 Hyphantis T, Palieraki K, Voulgari PV, Tsifetaki N, Drosos AA: Coping with healthstressors and defence styles associated with health-related quality of life in patients with systemic lupus erythematosus. Lupus 2011; 20:893-903. 